\title{
Framing 0/1
}

Wie die Medien über die „Digitalisierung der Gesellschaft" berichten

\section{Frauke Zeller / Jens Wolling / Pablo Porten-Cheé}

Die Art und Weise, wie die Medien über Technik und die damit verbundenen Risiken berichten, wird immer wieder problematisiert. An diese Debatte knüpft die vorliegende Studie an. Es wird untersucht, wie das Thema Digitalisierung und digitale Technologien in ausgewählten Printmedien im Zeitverlauf dargestellt wird. Die Ergebnisse der Untersuchung zeigen $u$. a., dass diese Technologien kaum in der Kritik stehen. Die empirischen Ergebnisse in zwei Zeiträumen sind zudem strukturell weitgehend identisch. Die wenigen Unterschiede, die festzustellen sind, deuten auf eine tendenzielle Eintrübung des Images digitaler Technologie hin. Nichtsdestotrotz wird von den Medien insgesamt ein eher positives Bild der Digitalisierung gezeichnet. Allerdings gilt dieses Bild nicht für alle Bereiche: Sowie nicht nur der eher konfliktfreie Unterhaltungsbereich im Mittelpunkt stebt, sondern politische Aspekte oder Fragen von Recht und Sicherbeit thematisiert werden, wandelt sich dieses Bild. Methodisch zeigte sich zudem, dass die Kombination von holistischer und rekonstruktiver Frame-Ermittlung ein vielversprechendes empirisches Vorgehen darstellt.

Schlagwörter: Frame-Analyse, Digitalisierung, Technikberichterstattung, Inhaltsanalyse, Methodenvergleich

\section{Einleitung}

Die Grundlage für die meisten rezenten Medieninnovationen ist die Digitalisierung. Diese Basisinnovation ist von so grundlegender und weitreichender Bedeutung, dass häufig sogar von einer Digitalisierung der Gesellschaft gesprochen wird (Negroponte 1995). Auch in einer aktuellen Delphi-Studie wird als Kernbotschaft hervorgehoben, dass „die Digitalisierung und die noch weiter zunehmende IKT-Durchdringung aller privaten und beruflichen Lebensbereiche“ unsere Gesellschaft in Zukunft noch stärker formen werden, als dies in der Gegenwart bereits der Fall ist (Kessel et al. 2009). Die Voraussetzung für die Durchsetzung von Medieninnovationen ist allerdings, dass die digitale Technologie grundsätzlich auf gesellschaftliche Akzeptanz stößt (Giesecke 2003). Kelly (2009) schreibt dazu: “[...] technology is adopted rarely on its merits alone; social forces both constrain and encourage adoption. Mass media is a significant influence, along with other social forces, in all stages of adoption" (S. 34).

Angesichts der kaum abschätzbaren gesellschaftlichen, ökonomischen und politischen Folgen, die mit der Implementierung digitaler Technologien verbunden sind, ist zu vermuten, dass in den Massenmedien eine intensive Diskussion und Auseinandersetzung mit dieser Entwicklung stattfinden; nicht zuletzt deswegen, weil die Medien selbst von der wachsenden Bedeutung der Digitalisierung stark betroffen sind. In diesem Zusammenhang fungieren die berichterstattenden Medien gleichzeitig sowohl als Spiegel der öffentlichen Meinung als auch als meinungsbildende Instanz. Durch die von ihnen aufgegriffenen Aspekte des Themas rahmen sie den soziotechnischen Prozess der Digitalisierung der Gesellschaft. Für die Durchsetzung und Akzeptanz von Technologien ist es daher von großer Bedeutung, wie sie in den Medien dargestellt werden. Dies dürfte 
insbesondere für jene Technologien gelten, mit denen der Nutzer unmittelbar in Kontakt tritt, wie es beispielsweise bei Medientechnologien der Fall ist.

Vor dem Hintergrund der häufig formulierten Behauptung, dass die deutsche (Medien-)Öffentlichkeit latent technikskeptisch sei (siehe hierzu z. B. Eikmann/Herr 2008), stellt sich die Frage, ob sich solche Tendenzen auch in der Berichterstattung über Digitalisierung zeigen. Dass die Folgen der Digitalisierung in der öffentlichen Debatte durchaus problematisiert werden, lässt sich anhand des medialen Diskurses über den sog. „Gläsernen Bürger“ zeigen: Hinsichtlich der Chancen eines wirksamen Datenschutzes im beruflichen und privaten Leben wird dort ein kritisches, wenn nicht sogar düsteres Bild gezeichnet. Einsatzszenarien digitaler Innovationen beispielsweise im Gesundheitswesen oder auch bei Google Street View sind aktuelle Beispiele hierfür. Dem gegenüber stehen aber Erfolgsgeschichten wie Facebook oder Twitter mit einer rasanten Durchsetzung in Wirtschaft, Politik und vielen Bereichen der Gesellschaft. Vorliegende Befunde zeigen zudem, dass zumindest in den 90er Jahren die Medien beispielsweise über das Internet überwiegend positiv berichtet haben (Rössler 2001, S. 59). Somit erscheint es beim Thema Digitalisierung ebenfalls möglich, dass die Chancen und positiven Folgen eine größere Beachtung finden, als dies bei anderen technischen Innovationen der Fall ist.

Welche Sichtweise tatsächlich überwiegt, wie die verschiedenen digitalen Technologien in den Medien dargestellt werden und welche Aspekte dabei eine besondere Rolle spielen, zeigen die Ergebnisse einer Inhaltsanalyse von Printmedien, die in diesem Beitrag vorgestellt werden. Dazu wurden die verschiedenen gesellschaftlichen Zusammenhänge, in denen digitale Technologie und speziell digitale Medientechnologie eine Rolle spielen, herausgearbeitet und deren Veränderung im Zeitverlauf aufgezeigt. Als konzeptionelle Basis für die empirische Untersuchung wurde der Framing-Ansatz gewählt: zum einen, weil bereits Erfahrungen mit dem Ansatz aus ähnlichen Gegenstandsbereichen der Technikberichterstattung vorliegen und er sich dort als gut anwendbar erwiesen hat, und zum anderen, weil er erlaubt, ein differenziertes Bild der medialen Darstellung des Themas zu zeichnen. Der Ansatz hat in den letzten Jahren in der kommunikationswissenschaftlichen Forschung zunehmende Beachtung erfahren und wurde in zahlreichen Studien als theoretische Grundlage gewählt (Matthes 2008 zählt allein in ausgewählten peer-reviewed Journals über 130 Beiträge zwischen 1990 und 2005). Trotz oder möglicherweise gerade wegen - dieser intensiven Nutzung des Framing-Ansatzes werden in der Literatur zahlreiche theoretische, konzeptionelle und forschungspraktische Probleme, die bei der Anwendung des Ansatzes zu lösen sind, intensiv debattiert. Aus diesem Grund wird im vorliegenden Beitrag neben der thematischen Zielsetzung auch ein methodisches Forschungsinteresse verfolgt. Ziel dieses Aufsatzes ist es deswegen, zwei Fragen zu beantworten, wobei die erste Frage inhaltlicher und die zweite methodischer Natur ist:

1. Wie wird über das Thema Digitalisierung berichtet und welche Veränderungen lassen sich zwischen den Jabren 2003 und 2008 feststellen?

2. Welche Unterschiede und Gemeinsamkeiten in den Ergebnissen sind auf spezifische Operationalisierungen von Medien-Frames zurückzufübren?

\section{Theorie: Technikberichterstattung und Framing}

Die Untersuchung der medialen Darstellung der Digitalisierung kann als Bestandteil der Forschung zur Technikkommunikation bzw. Technikberichterstattung eingeordnet werden. Kepplinger (1992) weist darauf hin, dass sich die Darstellung der Technik in den 
Medien seit Ende der 60er Jahre allmählich verändert hat. Während in den 50er und 60er Jahren zum einen generell wenig und zum anderen überwiegend positiv berichtet worden war, gab es danach eine zunehmend kritische Berichterstattung. Diese Umbewertung ging mit einer wachsenden medialen Thematisierung von Technik einher und erfasste mehr oder weniger alle Technikbereiche. Eine plausible Ursache für diese Umbewertung könnte die Wahrnehmung wachsender Technikschäden sein. Entsprechend stellt Kepplinger fest: „Zu den Gewissheiten gehört auch die Überzeugung, dass die Umbewertung der Technik in der Berichterstattung der Massenmedien eine Folge des geschärften Problembewusstseins der Bevölkerung ist, deren Ängste sie spiegelt“ (1992, S. 94). Kepplingers (1989) Ergebnisse zeigen jedoch, dass zwischen der Schwere der Technikschäden und der Art der Berichterstattung keine entsprechenden Zusammenhänge bestehen. Die veränderte Darstellung ist nach Kepplingers (1993) Ansicht vielmehr auf gesellschaftliche Veränderungen und ein verändertes Rollenverständnis der Journalisten zurückzuführen, die insgesamt zu einer stärker die Konflikte und die Risiken betonenden Technikberichterstattung geführt haben. Auch Dahinden und Schanne (2009) konstatieren, dass viele Technikdebatten politisiert und kontrovers geführt werden. Die möglichen Auswirkungen von insbesondere Bio-, Nano- und Energietechnologien auf Umwelt und Gesundheit stehen dabei häufig im Mittelpunkt. Die Technikberichterstattung wird deswegen in der wissenschaftlichen Debatte häufig unter dem Label der Risikokommunikation verhandelt. Es fällt allerdings auf, dass die Technikberichterstattung zur Digitalisierung bzw. zu digitalen Technologien in dieser Aufzählung nicht auftaucht. Dies kann sicherlich einerseits darin begründet sein, dass digitale Technologien kein klar abgegrenztes Gebiet darstellen, wie z. B. die Kernenergie. Andererseits sind digitale Technologien wie Computer oder Mobiltelefon direkter in das Alltagsleben integriert, wohingegen die Bereiche Kernenergie, Bio-, Nano- und Energietechnologie für den Einzelnen weniger greifbar sind. Aus der Umweltkommunikation ist bekannt, dass die Bedrohung der Umwelt im Nahbereich meistens geringer eingeschätzt wird als im Fernbereich (Hansen 1995). Möglicherweise liegen im Bereich digitaler Technologien ähnliche Zusammenhänge vor.

Durch die Fokussierung der Forschung zur Technikberichterstattung auf Risikotechnologien ist es nicht verwunderlich, dass mittlerweile eine Vielzahl an Studien zur Berichterstattung der Medien insbesondere über Kernenergie existieren (Maurer/Reinemann 2006), zu digitalen Technologien allerdings weniger Studien vorliegen. Vielmehr finden sich hier vereinzelte und fokussierte Studien, wie beispielsweise zur Darstellung des Internets durch die Medien (Rössler 2001) oder zur Multimedia-Berichterstattung (Beck/Vowe 1995). Trotz dieses einseitigen Fokus trifft man immer wieder auf die generalisierende Behauptung, dass die deutsche (Medien-)Öffentlichkeit technikskeptisch sei. Diese Aussage kann aber weder durch die nur partiell vorhandenen Untersuchungen zur Berichterstattung zu digitalen Technologien bestätigt werden, noch zeigen Studien aus dem Bereich der Technikfolgeabschätzung und Technikakzeptanz diesbezüglich eine eindeutige Tendenz. Diese vermitteln vielmehr ein Bild, das weder eindeutig in Richtung Technikakzeptanz noch Technikskepsis zeigt (Kessel et al. 2009).

Mit diesem Beitrag soll diese Unklarheit ein Stück weit abgeschafft werden, indem die Berichterstattung der Medien zum Thema Digitalisierung untersucht wird. Hierzu wird in Anlehnung an Dahinden und Schanne (2009) der Bereich Technikberichterstattung nicht nur als Teilgebiet der Wissenschafts- und Risikokommunikation verstanden, sondern es werden beispielsweise auch serviceorientierte Beiträge integriert. Operationalisiert wird dieser Ansatz durch den Rückgriff auf bereits bestehende Studien aus diesen Bereichen, wie der von Beck und Vowe (1995) entwickelte Framing-Ansatz zur 
Multimediaberichterstattung (bezüglich des Bereichs Technikberichterstattung). Ferner wird für den Bereich der Wissenschaftskommunikation die Frame-Unteruchung von Peters (1994) herangezogen sowie bezüglich der Risikokommunikation die Biotechnologie-Studie von Matthes und Kohring (2004).

Die Ermittlung der verschiedenen Berichterstattungszusammenhänge über das Thema Digitalisierung erfolgte auf Grundlage des Framing-Ansatzes. Der Ansatz wird allerdings bezüglich einer fehlenden einheitlichen Definition und vor allem eines fehlenden konkreten methodologischen Rahmens kritisiert ${ }^{1}$, was zumindest hinsichtlich der Vielzahl unterschiedlicher Operationalisierungen zutrifft.

Framing-Prozesse können auf vier unterschiedlichen Ebenen (levels) ermittelt werden: 1) in der Kultur, 2) in den Ansichten von Eliten und professionellen politischen Kommunikatoren, 3) in den Texten der Berichterstattung (Medien-Frames) und 4) in den Ansichten der einzelnen Bürgerinnen und Bürger (Entman et al. 2009, S. 176). Für die hier vorliegende Studie ist das Konzept der Medien-Frames relevant. Medien-Frames sind Ausdruck und Ergebnis von Arbeitsroutinen, die es den Journalisten ermöglichen, Information schnell einzuordnen und effizient weiterzuverarbeiten (Scheufele 1999).

Die durch das journalistische Handeln entstehenden Medien-Frames sind einerseits ganzheitliche kognitive Strukturen, anderseits bestehen sie jedoch aus einer Reihe von einzelnen Elementen. Dazu gehören im Anschluss an Entman (1993) insbesondere Problemdefinitionen und Themenaspekte, Ursachen- und Verantwortungszuschreibungen sowie Folgeabschätzungen. Diese Elemente sind in stabilen, identifizierbaren Mustern organisiert. Dadurch wird die ganzheitliche Wahrnehmung möglich. Medien-Frames können somit als mehrdimensionale Konfigurationen von Berichterstattungseigenschaften interpretiert werden, durch die eine bestimmte Sichtweise auf den jeweiligen Berichterstattungsgegenstand konstruiert wird (vgl. Scheufele 2003, S. 55ff.; Bonfadelli 2002, S. 143ff.). Durch eine solche Rahmung lassen sich bestimmte Realitätseinschätzungen fördern und andere zurückdrängen.

Auch wenn hinsichtlich dieses Grundverständnisses weitgehend Einigkeit herrscht, ist die Art und Weise, wie Medien-Frames empirisch zu erfassen sind, durchaus umstritten. Matthes (2008) gibt einen Überblick über die verschiedenen inhaltsanalytischen Herangehensweisen und Grundsatzentscheidungen sowie die unterschiedlichen Varianten der ermittelten Frame-Typen. Diese Aspekte sollen hier kurz vorgestellt werden, nicht zuletzt, um die Relevanz der methodischen Fragestellung der Studie deutlich zu machen.

Die erste grundlegende Unterscheidung bezieht sich auf die Frage, ob formal-stilistische, globale (themenübergreifende) oder themenspezifische Frames zu untersuchen sind. Für das hier vorliegende Forschungsinteresse sind themenspezifische Frames geeignet. Bei formal stilistischen Frames tritt der Inhalt der Botschaft in den Hintergrund, deswegen bieten sie für diese Arbeit keinen sinnvollen Ansatzpunkt. Im Hinblick auf die globalen Frames problematisiert Matthes (2008) deren zu hohes Abstraktionsniveau und unzureichende Trennschärfe sowie unklare Abgrenzung zu anderen Konstrukten, wie beispielsweise Themen oder auch Nachrichtenfaktoren. Dieser Argumentation wird im Rahmen dieses Projekts gefolgt.

Die zweite Unterscheidung bezieht sich auf den methodischen Zugang. Die empirische Untersuchung von Frames bzw. Framing in den Medien wird in qualitative und quantitative Herangehensweisen differenziert. Bei der qualitativen Herangehensweise

1 Für einen aktuellen Überblick zur Framing-Literatur siehe D’Angelo/Kuypers (2009), Entman et al. (2009), Matthes (2008). 
wird eine offene Inhaltsanalyse verwendet, die darauf abzielt, die in den Texten vorhandenen Frames induktiv herauszufiltern. Diese Form von Framing-Analyse dient vor allem der Exploration und ist entsprechend aufwändig. Eine größere Anzahl an Medienangeboten kann auf diese Weise kaum untersucht werden. Bei der quantitativen Herangehensweise werden zum einen computerbasierte und zum anderen „manuelle“, von Kodierern durchgeführte Analysen unterschieden. Bei der manuellen Analyse kommen wiederum zwei Varianten zum Einsatz. Beide erfordern eine deduktive Herangehensweise: Entweder werden als Ausgangspunkt verschiedene vordefinierte holistische Frames oder aber einzelne aus den Frame-Definitionen abgeleitete Elemente vorab festgelegt. Die inhaltsanalytischen Variablen sind im ersten Fall die kompletten Frames und im zweiten Fall die einzelnen Frame-Elemente, aus denen dann die Frames mit entsprechenden statistischen Verfahren ermittelt werden (dimensionsreduzierender bzw. rekonstruktiver Zugang). Beide deduktive Verfahren wurden in der vorliegenden Studie verwendet.

Zur Bestimmung der holistischen Frames wurde zum einen die Arbeit von Beck und Vowe (1995) zur Multimedia-Berichterstattung herangezogen. Hiernach können vier als Frames interpretierbare Sichtweisen auf das Thema identifiziert werden, die von den Autoren als die Maschinenperspektive, die Medienperspektive, die Warenperspektive und die Steuerungsperspektive bezeichnet wurden. Zum anderen wurden die Ergebnisse einer Studie von Peters zur Rolle von Experten in der Wissenschaftskommunikation (1994) genutzt. Er unterscheidet ebenfalls vier Frames, die er als PopularisierungsFrame, Orientierungs-Frame, Kontroverse-Frame und Skandal-Frame bezeichnet. Diese acht auf den Untersuchungsgegenstand zugeschnittenen, theoretisch fundierten und empirisch erhärteten Frames wurden für die vorliegende Untersuchung nutzbar gemacht und operationalisiert. Dabei wurden sie - soweit notwendig - an den Untersuchungsgegenstand angepasst, da in den beiden Studien Frames sehr unterschiedlich konzeptionalisiert werden. Während Peters nur ermittelt, ob der jeweilige Frame vorhanden ist oder nicht, unterscheiden Beck und Vowe zusätzlich auch deren Wertigkeit, indem sie jeweils einen positiven und einen negativen Pol der Frames definieren. Diese unterschiedlichen Konzeptionalisierungen wurden beibehalten.

$\mathrm{Ob}$ es möglich ist, solche holistischen Medien-Frames reliabel und valide zu erfassen, wird jedoch immer wieder in Frage gestellt (vgl. Matthes/Kohring 2004). Scheufele und Scheufele (2009) argumentieren, dass mit der Verwendung vordefinierter Frames eher die kognitiven Frames der Forscher als die tatsächlich in den Medien vorhandenen Frames erfasst werden. Matthes und Kohring (2004, S. 61) bieten daher eine Methode an, welche die Validität und die objektive Erfassung von Medien-Frames sicherstellen soll. Hierzu greifen sie die Frame-Definition von Entman auf und schlagen vor, nicht den kompletten Frame zu kodieren, sondern die einzelnen Elemente: Problemdefinition, kausale Interpretation, moralische Bewertung und Handlungsempfehlungen (ebd.). Grundannahme dieses Verfahrens ist es, Frames als konkrete unverwechselbare Muster eines Texts zu begreifen, die „sich aus mehreren, von Journalisten selektiv ausgewählten und von Rezipienten beobachtbaren Elementen zusammensetz[en]“ (ebd., S. 61). Nach dieser Argumentation sind diese Muster anhand der Einzelelemente empirisch bestimmbar.

In der hier dargestellten Untersuchung wurden die beiden in der aktuellen FramingLiteratur kontrovers diskutierten deduktiven Herangehensweisen kombiniert, um ihre „Leistungsfähigkeit“ zu vergleichen. Eine solche Vorgehensweise war in diesem Fall problemlos möglich: zum einen, weil für die Auswahl der holistischen Frames auf Indikatoren zurückgegriffen werden konnte, die in thematisch verwandten Gegenstands- 
bereichen entwickelt (Beck/Vowe 1995, Peters 1994) und zudem bereits empirisch getestet worden waren (Rössler 2001); zum anderen, weil mit der Bezugnahme auf die Frame-Definition von Entman (1993) auch die Elemente für den rekonstruktiven $\mathrm{Zu-}$ gang im Wesentlichen deduziert werden konnten. Hierbei handelt es sich um folgende Elemente: thematische Einordnung, Problemdefinition, Prognose, Handlungsempfehlungen und Verantwortungszuschreibung für Schäden und Nutzen, sowie als Ergänzung zu gängigen operationalen Definitionen auch die Ermittlung von Begünstigten und Geschädigten der Digitalisierung.

Durch die Kombination dieser beiden Herangehensweisen wird zudem noch ein weiterer umstrittener Aspekt in der Framing-Forschung aufgegriffen: die Frage, ob in einem Beitrag nur ein oder aber mehrere Frames vorhanden sein können (Matthes 2008). Für beide Herangehensweisen gibt es plausible Argumente, deswegen wurden beide Optionen integriert. Bei der Codierung der holistischen Frames bestand die Möglichkeit, mehrere Frames zu kodieren, bei der rekonstruktiven Vorgehensweise hingegen wurde nur ein Frame pro Beitrag ermittelt.

Ein weiteres wichtiges Element des Ansatzes ist der schon bei Entman vorhandene Fokus auf den diachronischen Charakter, der allerdings in den meisten Framing-Studien kaum Beachtung fand (Matthes 2008). Framing erfolgt dadurch, dass gleiche Objekte immer wieder mit gleichen oder ähnlichen Eigenschaften in unterschiedlichen Zusammenhängen wiederholt werden: “A frame repeatedly invokes the same objects and traits, using identical or synonymous words and symbols in a series of similar communications that are concentrated in time. These frames function to promote an interpretation of a problematic situation or actor and (implicit or explicit) support of a desirable response, often along with a moral judgement that provides an emotional charge" (Entman et al. 2009, S. 177). Framing erfolgt im Zeitverlauf und ist durch sich selbst verstärkende Prozesse geprägt: "Framing is thus diachronic in the sense that exposure during a given period is presumed to increase probabilities of particular responses during a future period, while diminishing the probability of thinking about other potentially relevant objects or traits“ (ebd.). Dies trifft insbesondere auch auf die Thematik der medialen Darstellung der Digitalisierung in der Gesellschaft zu, da die gesellschaftliche Akzeptanz digitaler Technologien einer prozessualen Entwicklung unterworfen ist. Deswegen wird im Rahmen dieser Untersuchung die Darstellung digitaler Technologien in den Medien im Zeitvergleich untersucht. So können einerseits sich wiederholende Frames in einem zusammenhängenden Zeitraum und andererseits auch Frame-Veränderungen in einem größeren Zeitabstand aufgedeckt werden. Von besonderem Interesse ist dabei, ob sich einheitliche Entwicklungstendenzen feststellen lassen oder ob sich möglicherweise gegenläufige Prozesse in der Darstellung der Digitalisierung vollziehen, da nicht anzunehmen ist, dass sich jede digitale Medieninnovation in ein generelles Bild einfügt.

\section{Methode}

Zur Beantwortung der Forschungsfrage wurde als Methode eine quantitative Inhaltsanalyse gewählt, mit der die im Theorie-Abschnitt diskutierten unterschiedlichen Framing-Ansätze erhoben wurden. Analysiert wurden überregionale deutsche Tageszeitungen („Süddeutsche Zeitung“, „Frankfurter Allgemeine Zeitung“ und „die tageszeitung“) und Zeitschriften („Der Spiegel“, „Focus“ und „Stern“). Die Untersuchung wurde in zwei separaten Zeiträumen in einem Abstand von fünf Jahren durchgeführt: Juli bis Dezember 2003 und Juli bis Dezember 2008.

Die Auswahl sollte dem Leitcharakter dieser Printmedien in Deutschland Rechnung tragen und darüber hinaus die redaktionellen Links-Rechts-Tendenzen abdecken (vgl. 
Noelle-Neumann et al. 1999). Als Analyseeinheiten wurden Artikel ausgewählt, in denen das Wort „digital“ als solches oder als Wortbestandteil vorkam. In einem zweiten Auswahlschritt wurden aus dieser Beitragssammlung nur solche Artikel berücksichtigt, in denen Digitalisierung bzw. digitale Technologien eine nennenswerte Rolle spielten. Die aus der Datenbank „Lexis Nexis“ gezogene Stichprobe (mit Ausnahme der Süddeutschen Zeitung - hier wurde eine Offline-Datenbank genutzt) umfasst 421 Artikel 210 für das zweite Halbjahr 2003 und 211 für das zweite Halbjahr 2008. Die relevante Berichterstattung der drei Zeitschriften wurde vollständig analysiert (Vollerhebung), bei den drei Tageszeitungen wurde jeweils eine Stichprobe von 55 Prozent gezogen. ${ }^{2}$ Die annähernd identischen Fallzahlen in den beiden Jahren zeigen, dass der Umfang der Berichterstattung in den beiden Erhebungszeiträumen nahezu gleich geblieben ist. Eine typische Themenkarriere für Technikthemen, wie von Kolb (2005) am Beispiel Blei im Benzin ermittelt, findet sich hier nicht. Das entspricht dem Ergebnis von Rössler (2001) für die Berichterstattung zum Thema Internet, auch er findet eine eher kontinuierliche Thematisierung.

Die Entwicklung des Kodebuchs ${ }^{3}$ wurde im Rahmen eines Master-Seminars gemeinsam mit Studierenden vorgenommen. Die Operationalisierung der Frames erfolgte wie in den Tabellen 1 und 2 beschrieben. Bei den Probekodierungen zeigte sich, dass bei der Kodierung des Frame-Elements „moralische Bewertung“ keine reliable Messung zu erzielen war. Auf dieses Frame-Element wurde somit verzichtet und in Anlehnung an Matthes und Kohring (2004, S. 64) die Variable „Prognose“ eingeführt. Diese erfasst auch moralische Aspekte, da sie eine Bewertung beinhaltet, wie sich ein zu erwartender $\mathrm{Zu}-$ stand zu gegenwärtigen und fortdauernden Normen verhält.

Somit ergaben sich die vier Frame-Elemente „Problemdefinition“, „kausale Interpretation“, „Prognose“ und „Handlungsempfehlungen“, wobei das Element „Problemdefinition“ a) aus den Hauptthemen der Berichterstattung, b) der Schaden-Nutzenbewertung des Hauptthemas und c) der Erfassung, wer in dem Zusammenhang geschädigt bzw. begünstigt wird, besteht. Die Hauptthemen der Berichterstattung wurden auf Basis früherer Inhaltsanalysen konzipiert. Sie sind allgemeiner formuliert als beispielsweise bei der Erhebung der Berichterstattung über Biotechnologie (Matthes/Kohring 2004), da zu „Digitalisierung“ eine größere thematische Heterogenität erwartet wurde. Das Frame-Element „kausale Interpretation“ beinhaltet den Verantwortlichen für den thematisierten Schaden bzw. Nutzen. Die Handlungsempfehlungen wurden im Unterschied zu Matthes und Kohring wiederum vereinfacht und nicht die Art der Handlungsempfehlung, sondern nur ihr Vorhandensein erhoben. Ursprünglich nominal-skalierte Variablen (Themen, Verantwortliche, Begünstigte, Geschädigte) oder ordinal-skalierte Variablen (Schaden-Nutzen-Dimension und Bewertung des Themas, Prognose) wurden zur besseren Vergleichbarkeit bei nachfolgenden deskriptiven Darstellungen in eine 0/1-Skala (nicht genannt/genannt) standardisiert.

Zusätzlich zu den Frame-Elementen beinhaltete das Kodebuch noch die oben erwähnten vier holistischen Frame-Variablen nach Beck und Vowe (1995). Dabei wurden

2 Wie anschließende deskriptive Analysen belegten, besaß dieser Umstand keine Konsequenzen für die Interpretation der Daten. Es ließen sich zwar in Abhängigkeit der Mediengattung leicht unterschiedliche Framing-Modi feststellen, diese Beobachtung widersprach aber nicht dem Gesamtbild, nach dem in Tageszeitungen und Zeitschriften identische Muster repräsentiert waren. Deswegen wurde auf eine Nachgewichtung verzichtet.

3 Das vollständige Kodebuch ist einsehbar unter: www.tu-ilmenau.de/empk/forschung/digitali sierung-in-den-medien/. Für die Publikation wurde nur ein Teil der Variablen verwendet und diese zum Teil für die Publikation rekodiert. 
Tabelle 1: Operationalisierung der Frame-Elemente nach Matthes und Kohring (2004)

\begin{tabular}{|c|c|c|c|c|}
\hline \multirow{2}{*}{$\begin{array}{c}\text { Frame- } \\
\text { Elemente } \\
\text { nach Entman } \\
(1993) \\
\end{array}$} & \multicolumn{2}{|c|}{$\begin{array}{l}\text { Operationalisierung nach Matthes } \\
\text { und Kohring (2004) }\end{array}$} & \multicolumn{2}{|c|}{$\begin{array}{c}\text { Operationalisierung im Kontext einer } \\
\text { Berichterstattung über Digitalisierung und } \\
\text { Gesellschaft }\end{array}$} \\
\hline & Kategorien & $\begin{array}{c}\text { Skala } \\
\text { (dichotom) }\end{array}$ & Kategorien & Skala (dichotom) \\
\hline \multirow{3}{*}{$\begin{array}{l}\text { Problem- } \\
\text { definition }\end{array}$} & \multirow[t]{2}{*}{$\begin{array}{l}\text { Hauptthemen in } \\
\text { Bezug auf Bio- } \\
\text { technologie (Bsp.: } \\
\text { Landwirtschaft, } \\
\text { Forschung) }\end{array}$} & \multirow{4}{*}{$\begin{array}{l}\text { nicht } \\
\text { vorhanden/ } \\
\text { vorhanden }\end{array}$} & $\begin{array}{l}\text { Thema: Kultur, } \\
\text { Unterhaltung \& Freizeit } \\
\text { Thema: Wirtschaft } \\
\text { Thema: Recht und } \\
\text { Sicherheit } \\
\text { Sonstige Themen }\end{array}$ & $\begin{array}{c}0, \text {,nicht vorhanden“/ } \\
1 \text { „,vorhanden“ }\end{array}$ \\
\hline & & & $\begin{array}{l}\text { zusätzlich: Schaden oder } \\
\text { Nutzen thematisiert } \\
\text { zusätzlich: Bewertung des } \\
\text { Themas insgesamt }\end{array}$ & $\begin{array}{l}\text { Mittelwertsindex von } 0 \\
\text { „Schaden thematisiert, } \\
\text { negativ bewertet“ bis } 1 \\
\text { "Nutzen thematisiert, } \\
\text { positiv bewertet“" }\end{array}$ \\
\hline & $\begin{array}{l}\text { Nutzen für einzelne } \\
\text { Gesellschaftsbereiche } \\
\text { (Bsp.: Wirtschaft, } \\
\text { Medizin) }\end{array}$ & & $\begin{array}{l}\text { Begünstiger bzw. } \\
\text { Geschädigter: } \\
\text { - Wirtschaft } \\
\text { - Gesellschaft }\end{array}$ & $\begin{array}{l}0 \text {,nicht vorhanden“/ } \\
1 \text {,,vorhanden“ }\end{array}$ \\
\hline $\begin{array}{c}\text { Kausale } \\
\text { Interpretation }\end{array}$ & $\begin{array}{l}\text { Verantwortlicher } \\
\text { Akteur für Nutzen } \\
\text { bzw. Schaden }\end{array}$ & & $\begin{array}{c}\text { Verantwortlicher f. } \\
\text { Nutzen bzw. Schaden: } \\
\text { - Politik } \\
\text { - Wirtschaft } \\
\text { - Medien \& Gesellschaft }\end{array}$ & $\begin{array}{c}0 \text {,nicht vorhanden“/ } \\
1 \text {,,vorhanden“ }\end{array}$ \\
\hline $\begin{array}{l}\text { Moralische } \\
\text { Bewertung }\end{array}$ & $\begin{array}{l}\text { Zukünftige } \\
\text { Akzeptanz }\end{array}$ & $\begin{array}{l}\text { positive vs. } \\
\text { negative } \\
\text { Akzeptanz }\end{array}$ & \multicolumn{2}{|c|}{ nicht verwendet } \\
\hline $\begin{array}{l}\text { Handlungs- } \\
\text { empfehlung }\end{array}$ & $\begin{array}{l}\text { Aufforderung zur } \\
\text { Ausführung bzw. } \\
\text { Unterlassung } \\
\\
\text { positive bzw. } \\
\text { negative Prognose }\end{array}$ & $\begin{array}{l}\text { Ausführung } \\
\text { bzw. } \\
\text { Unterlassung } \\
\text { positive vs. } \\
\text { negative } \\
\text { Prognose }\end{array}$ & Handlungsempfehlung & $\begin{array}{c}0, \text {,nicht vorhanden“/ } \\
1, \text {,vorhanden“ } \\
0 \text {,negative Prognose“/ } \\
1 \text {,positive Prognose“ }\end{array}$ \\
\hline
\end{tabular}

die von Beck und Vowe ermittelten Frames dem Kontext der Berichterstattung über Digitalisierung und Gesellschaft angepasst. Hierzu wurde induktiv vorgegangen, indem die nach Kepplinger (1989) relevanten Nutzungsfelder von Technik unter Bezugnahme auf die Ressorts/Rubriken definiert wurden, in denen die meisten Beiträge zu Digitalisierungsthemen in den Printmedien vorkommen. Dieser Vorgang ergab die folgenden Frames: Technik-Frame, soziologischer/sozialpsychologischer Frame (nachfolgend Gesellschafts-Frame), Ökonomie-Frame sowie Politik-Frame. Die Frame-Auswahl zeigt wiederum, dass das Thema Digitalisierung kein fest abgrenzbares Gebiet ist, sondern viele Produkte, Serviceleistungen und Verfahren integriert und alle Bereiche der Gesellschaft betreffen kann. Neben diesen vier Frames kamen die von Peters (1994) übernommenen Frame-Variablen Popularisierungs-Frame, Orientierungs-Frame, Kontroverseund Skandalframe hinzu. Mit dieser Herangehensweise soll die Berichterstattung zur 
Tabelle 2: Operationalisierung der holistischen Frames nach Beck/Vowe (1995) und Peters (1994)

Holistische Frames

Nach Beck und Vowe (1995)

Skala

Bezeichnung

Maschinenperspektive

Medienperspektive

Warenperspektive (dichotom)

Bezeichnung und Kurzbeschreibung

Technik-Frame

Fokus auf Innovativität in der digitalen Entwicklung (Bsp.: technische Evolutionen wie die Erfindung des mp3-Formats)

Gesellschafts-Frame

0 ,nicht vorhanden“

Fokus auf gesellschaftliche Folgen der Digitalisierung (Bsp.: Folgen von Web 2.0 für

positiv dargestellt/ negativ dargestellt

die interpersonale Kommunikation)

\section{Ökonomie-Frame}

1, ,positiv dargestellt“

2 ,positiv und negativ dargestellt"

Fokus auf den ökonom. Wachstumscharakter

der Digitalisierung (Bsp.: Marktimpulse durch 3 ,,negativ dargestellt“ neue Online-Vertriebskanäle)

\section{Politik-Frame}

Fokus auf Digitalisierung als Gegenstand politischer Debatte (Bsp.: staatlich geförderte

DSL-Abdeckung im ländlichen Raum)

Nach Peters (1994)

Skala

Frame (dichotom)

Bezeichnung und Kurzbeschreibung

Skala (dichotom)

analog Peters (1994)

Popularisierungs-Frame

Orientierungs-Frame

Skandal-Frame

Einseitige positive Darstellung digitaler Innovationen (Bsp.: Einführung einer neuen digitalen Spiegel-Reflex-Kamera)

analog Peters (1994)

Digitalisierung wird als praktisches Problem behandelt (Bsp.: Datensicherheit im "Cloud Computing")

analog Peters (1994)

0 ,nicht vorhanden“/ 1 ,,vorhanden“"

Unstimmigkeiten in Bezug auf Digitalisierung

(Bsp.: Pro und Contra Einführung des digitalen Personalausweises)

analog Peters (1994)

Täter-Opfer-Ereignis in Verbindung mit dem Thema Digitalisierung (Bsp.: HackerAttacken)

Digitalisierung nicht ausschließlich - wie sonst bei der Erforschung der Technikkommunikation häufig üblich - als ein Teilgebiet der Risiko- und Wissenskommunikation eingeordnet werden, sondern eine pragmatische Annäherung an die unterschiedlichen 
Bereiche der Digitalisierung vorgenommen werden. Dies entspricht auch Kepplingers (1989) Feststellung, dass eine „umfassende Analyse der Darstellung von Technik nicht auf einzelne Produkte oder Verfahren beschränkt sein darf“ (S. 16).

Insgesamt besteht das Kodebuch aus 63 Variablen. Die Kodierung wurde von insgesamt 11 geschulten studentischen Kodierern durchgeführt. Das Vorgehen sah vor, dass ein Artikel jeweils von zwei Personen unabhängig voneinander kodiert wurde, wobei die Kodiererpaare wechselten, sodass jeder Kodierer mit jedem anderen einige Artikel gemeinsam zu kodieren hatte. Die finale Kodierung war das Ergebnis eines anschließenden Ergebnisvergleichs der zwei Kodierer, die sich bei Abweichungen auf eine Kodierung einigen mussten. Auf diese Weise konnte im formellen Sinne jeweils eine perfekte Intercoder-Reliabilität erreicht werden.

Die nachfolgend vorgestellten Befunde zu den aus Einzelelementen rekonstruierten Frames wurden mittels einer explorativ angelegten, hierarchischen Clusteranalyse ermittelt. Primäres Vorhaben clusteranalytischer Auswertungsverfahren ist die Zusammenfassung von Klassifikationsobjekten in homogene Gruppen (Klassen, Cluster, Typen). Ziel ist das „Auffinden einer empirischen Klassifikation (Gruppeneinteilung, Typologie)“ (Bacher 2002, S. 1). Das Ziel in dieser Untersuchung war die Bildung von Frame-Clustern auf Basis der Frame-Elemente Problemdefinition, kausale Interpretation, Handlungsempfehlung und Prognose. Für die Clusteranalyse wurden 17 Einzelvariablen ${ }^{4}$ herangezogen, anhand derer die Artikel gruppiert werden. Auf diese Weise wurden Cluster ermittelt, in denen jeweils Beiträge, deren Merkmale sehr ähnlich sind, zusammengefasst wurden (Grundprinzip der Homogenität bzw. interne Kohäsion) und die sich gleichzeitig hinsichtlich dieser Merkmale von anderen Clustern möglichst stark unterscheiden (Prinzip der Heterogenität bzw. externe Isolierung). Die Messung der Ähnlichkeiten von Fällen (Artikeln) anhand dichotomer und intervall-skalierter Variablen erfolgte durch das Distanzmaß des quadrierten euklidischen Abstands. Fälle sind sich demnach ähnlicher, je kleiner dieses Distanzmaß ist (Backhaus et al. 2006, S. 502ff.).

Als Fusionierungsalgorithmus wurde das Ward-Verfahren gewählt, welches in der Praxis weit verbreitet ist und im Vergleich zu anderen Algorithmen eine gut interpretierbare Gruppierung von Fällen vollzieht (ebd., S. 528). Das Ward-Verfahren dient der Bildung möglichst homogener Cluster, indem jeweils diejenigen Objekte (Gruppen) vereinigt werden, welche die Streuung (Varianz) in einer Gruppe möglichst wenig erhöhen (ebd., S. 522). Führt die Bildung eines neuen Clusters dazu, dass die Homogenität innerhalb der Cluster geringer wird bzw. eine sprunghafte Erhöhung des Heterogenitätsmaßes zu beobachten ist, zeigt das sog. Elbow-Kriterium an, dass die optimale Clusteranzahl erreicht ist (ebd., S. 534). Für die Untersuchung von Veränderungen im Jahresvergleich schlagen Kohring und Matthes (2002, S. 148) vor, Clusteranalysen für jeden Untersuchungszeitpunkt einzeln durchzuführen. Beim Thema Digitalisierung zeigte sich jedoch, dass innerhalb des fünfjährigen Untersuchungsintervalls von grundsätzlich stabilen Frames ausgegangen werden kann, die sich nur graduell verändern. Deswegen

4 Die Variable Hauptthema „Information und Kommunikation“ wurde von der abschließenden Clusteranalyse ausgeschlossen, da sie in allen Clustern fast gleichstark ausgeprägt war und so nicht zu ihrer Differenzierung beitrug. 
werden im Folgenden die Ergebnisse der Clusteranalyse mit dem Gesamtsample dargestellt. ${ }^{5}$

\section{Ergebnisse}

\subsection{Clusteranalytisch ermittelte Frames}

Die Clusteranalyse ergab eine gut interpretierbare 5-Cluster-Lösung (Tabelle 3). Das größte Cluster umfasst mit 164 Fällen knapp 40 Prozent des Gesamtsamples. Es ist thematisch durch den Bereich Kultur, Unterbaltung und Freizeit geprägt. Besonders auffällig sind zum einen der hohe Wert bei der Problemdefinition, der auf eine positive Bewertung des mit dem Thema verbundenen Nutzens hinweist, und zum anderen die sehr häufige Nennung der Gesellschaft als einen durch digitale Technologie begünstigten Akteur. Die Urheber des Nutzens sind in verschiedenen Gruppen zu verorten. Schaden hingegen spielt bei den Beiträgen in diesem Cluster keine Rolle. Insgesamt kommt in den Beiträgen dieses Clusters eine positive Bewertung der Digitalisierung zum Ausdruck. Insbesondere im Bereich Kultur, Unterhaltung und Freizeit stiftet sie für die Bürger und die Gesellschaft vor allem Nutzen und richtet keinen Schaden an. Ein typischer Artikel in diesem Cluster ist „Beim Spielen gut aussehen“ aus der Süddeutschen Zeitung (2008): Hier wird geschildert, wie die Berliner Philharmoniker „Mediengeschichte schreiben“, indem ihre Konzerte weltweit via DSL in der „Digital Concert Hall“ auch online abrufbar sein sollen. Wie in diesem Beispiel wird generell in diesem Cluster der Nutzen der digitalen Technologie hervorgehoben. Vor diesem Hintergrund wurde das Cluster als „The Bright Side“ der Digitalisierung bezeichnet.

Das zweitgrößte Cluster umfasst 112 Fälle und vereinigt damit knapp 27 Prozent aller Artikel. Im Mittelpunkt stehen bei den Beiträgen dieses Clusters Fragen von Recht und Sicherheit. Im deutlichen Unterschied zum ersten Cluster kommen hier besonders häufig Schäden zur Sprache. Vor allem die Bürger und die Gesellschaft werden als Geschädigte benannt. Allerdings werden in den Beiträgen auch Gewinner erwähnt, wenn auch deutlich weniger. Insgesamt überwiegt in den Beiträgen eine starke Problematisierung der Technologie, verbunden mit klaren Handlungsempfehlungen. Aufgrund der eher negativen Einschätzungen, die in den Beiträgen dieser Gruppe zum Ausdruck kommen, und des Aufzeigens von Problemfeldern der Digitalisierung wurde das Cluster als „The Dark Side“ der Digitalisierung bezeichnet. Ein typischer Artikel in diesem Cluster ist „Feinbild der Fans“ aus dem Wochenmagazin Der Spiegel (2003), bei dem die negative Botschaft bereits in der Überschrift zum Ausdruck kommt. Konkret handelt es sich bei dem Beitrag um ein Interview mit dem Sänger Marius Müller-Westernhagen, welcher sich zur digitalen Piraterie und dem „schleichenden Wertverlust der Ware Musik“ äußert und meint, dass die Piraterie das „Ergebnis einer technologischen Entwicklung [sei], die seit vielen Jahren bekannt ist“. Hier findet sich demnach das Thema Recht

5 Es wurden auch Clusteranalysen auf Basis der einzelnen Jahre durchgeführt. Diese boten ein ähnliches Frame-Muster wie in der Gesamtbetrachtung dar. Allerdings war diese Lösung auf Grund einer unterschiedlichen Clusteranzahl im Jahresvergleich weniger eindeutig zu interpretieren, weswegen der konsistenteren Gesamtbetrachtung der Vorzug gegeben wurde. Auch die gewählte Vorgehensweise lässt die Möglichkeit zu, dass bestimmte Frame-Cluster im Jahresvergleich unterschiedlich groß (Fallzahlen) ausfallen und unterschiedliche Eigenschaften (Variablenmittelwerte der Clustervariablen) aufweisen. Die ermittelten Cluster können entweder anwachsen, schrumpfen oder marginalisieren (vgl. zur zeitlichen Veränderbarkeit von Frames bspw. Scheufele/Brosius 1999). Als Nachteil dieser Strategie ist anzumerken, dass damit etwaige Fusionen oder Abspaltungen von Clustern nicht beobachtbar sind. 
Tabelle 3: Beschreibung der Frame-Cluster

\begin{tabular}{|l|l|l|}
\hline $\begin{array}{l}\text { Cluster der Frame- } \\
\text { Elemente }\end{array}$ & \multicolumn{1}{|c|}{$\begin{array}{c}\text { Größe } \\
\text { (Gesamt: } \mathbf{n = 4 2 1 )}\end{array}$} & Artikelbeispiel \\
\hline $\begin{array}{l}\text { „The Bright Side“der } \\
\text { Digitalisierung }\end{array}$ & $\mathrm{n}=164$ & $\begin{array}{l}\text { Online-Konzert der Berliner } \\
\text { Philharmoniker }\end{array}$ \\
\hline $\begin{array}{l}\text { „The Dark Side“ der } \\
\text { Digitalisierung }\end{array}$ & $\mathrm{n}=112$ & $\begin{array}{l}\text { Wertverlust der Musik durch } \\
\text { Piraterie }\end{array}$ \\
\hline $\begin{array}{l}\text { Wirtschaftlicher } \\
\text { Wettbewerb }\end{array}$ & $\mathrm{n}=79$ & $\begin{array}{l}\text { Beschreibung des Markts für } \\
\text { Musik-Börsen online }\end{array}$ \\
\hline $\begin{array}{l}\text { Gesamtgesellschaftlicher } \\
\text { Fortschritt }\end{array}$ & $\mathrm{n}=37$ & $\begin{array}{l}\text { Ältere Menschen profitieren vom } \\
\text { elektronischen Gesundheits-Check }\end{array}$ \\
\hline $\begin{array}{l}\text { Konsequenzen politischen } \\
\text { Handelns }\end{array}$ & $\mathrm{n}=29$ & Internet-Zensur in China \\
\hline
\end{tabular}

und Sicherheit als ein Schwerpunkt wieder, in dessen Rahmen digitale Technologien stark problematisiert werden.

Im drittgrößten Cluster steht das Thema Wirtschaft im Mittelpunkt. Das Cluster besteht aus 79 Fällen, das sind knapp 19 Prozent des Samples. Im Unterschied zu den beiden vorhergehenden Clustern ist das Verhältnis von Schaden und Nutzen ausgeglichen. Es findet weder eine starke Problematisierung noch eine einseitige Betonung von Chancen statt. Geprägt ist das Cluster in jeder Hinsicht durch das Thema Wirtschaft: Sowohl die Urheber als auch die Begünstigten sind Wirtschaftsakteure und dies sowohl im Hinblick auf nachteilige Auswirkungen der Technologie als auch auf Vorteile, die durch digitale Technologie entstehen. Ein typischer Artikel in diesem Cluster beschreibt den Neustart von Napster 2 in den USA (die tageszeitung, 2003). Napster war lange Zeit das Synonym für Filesharing, eine der grundlegenden neuen Möglichkeiten des Internets mit ebenso tiefgreifenden Folgen. Im Artikel geht es um die Beschreibung des neuen Geschäftsmodells bzw. Service-Angebots von Napster 2 und um den Vergleich mit anderen Wettbewerbern im Markt der Online-Musik-Börsen. Somit werden Vorteile und Nachteile dargestellt sowie unterschiedliche Wirtschaftsakteure als Begünstigte der Digitalisierung. Das Cluster wurde daher Wirtschaftlicher Wettbewerb genannt.

Cluster vier und fünf haben mit Abstand die geringsten Fallzahlen. Cluster vier besteht aus insgesamt 37 Fällen, also circa 9 Prozent des Samples. Hier finden sich Beiträge zu den Unterthemen Verkehr und Energie, Medizin, Naturwissenschaften (als „Thema: Sonstiges" zusammengefasst). Da dies alles Themen sind, welche unmittelbar die Gesellschaft betreffen, kann hier von einem gesellschaftlichen Fokus gesprochen werden. Auffallend ist, dass die gesellschaftlichen Akteure sehr viel häufiger als Begünstigte der Digitalisierung genannt werden als im Zusammenhang mit Schäden bzw. als Geschädigte. Mit Blick auf die Unterthemenkomplexe - von denen mindestens zwei eindeutig Forschung und Entwicklung ansprechen - entsteht das Bild eines gesamtgesellschaftlichen Fortschritts durch Digitalisierung, wie letztendlich auch das Cluster genannt wurde. Auch ein für dieses Cluster typischer Artikel bestätigt diese Entscheidung: In einem Bericht zum elektronischen Gesundheits-Check (FAZ, 2008) wird der Fortschritt auf dem Gebiet der digitalen Gesundheitsvorsorge am Beispiel eines neu entwickelten digitalen Systems erörtert, welches es ermöglicht, dass ältere Menschen länger alleine zu Hause leben können. Mit Hilfe des Systems könne heute schon die regelmäßige Medi- 
kamenteneinnahme fern-registriert werden, und in naher Zukunft solle auch der Gang älterer Menschen fern-überwacht werden können, so dass rechtzeitig vor drohenden Stürzen gewarnt werden könne.

Das letzte Cluster (Cluster fünf) schlägt mit gerade einmal 29 Fällen zu Buche, was letztendlich knappe 7 Prozent des Samples ausmacht. Hier findet sich ein stark ausgeprägter Politik-Fokus. Politische Akteure spielen sowohl als Verantwortliche für den Nutzen als auch für den Schaden eine wesentliche Rolle. Bezüglich der Geschädigten wird hier die Gesellschaft besonders häufig genannt, dicht gefolgt von der Wirtschaft. Ein kennzeichnender Artikel aus diesem Cluster beschreibt die Internet-Zensur in China seitens der chinesischen Regierung (Focus Magazin, 2008), womit die chinesische Regierung klar die Rolle des Verantwortlichen für Schaden, aber auch (Eigen-)Nutzen einnimmt. Als Folge daraus wird u. a. die Inhaftierung von Freiheitskämpferinnen dargestellt. Hieran werden die problematischen gesellschaftlichen Konsequenzen deutlich. Darüber hinaus spielt in diesem Beitrag aber auch die Wirtschaft eine ambivalente Rolle, da Unternehmen wie Google, Yahoo oder Microsoft genannt werden, welche die Zensur-Bemühungen der chinesischen Regierung aktiv unterstützen. Da in diesem Cluster beim Thema Politik auch die Akteurs-Dimension eine wichtige Rolle spielt, wurde das fünfte Cluster Konsequenzen politischen Handelns genannt.

\subsection{Zeitliche Veränderung der clusteranalytisch ermittelte Frames}

Die Clusteranalyse wurde - wie bereits oben erläutert - mit dem Gesamtsample durchgeführt. Anschließend wurde geprüft, ob sich die fünf Cluster in beiden Teilstichproben finden lassen. Dabei wurde zum einen untersucht, ob sich Veränderungen in der Bedeutung der Cluster beobachten lassen (Abbildung 1) und ob interne Verschiebungen in der Zusammensetzung der Cluster festzustellen sind (Delta in Tabelle 4).

Die Balkengrafik in Abbildung 1 verdeutlicht, dass sich die Cluster-Struktur zu beiden Zeitpunkten nur graduell unterscheidet. Diese hohe Stabilität belegt auch ein ChiQuadrat-Test $\left(\chi^{2}=3,25, \mathrm{p}=.52, \mathrm{df}=4\right)$. Die deutlichste Veränderung ist beim größten Cluster zu beobachten: „The Bright Side“ der Digitalisierung hat in der Berichterstattung an Bedeutung verloren. Allerdings ist dieses Cluster auch 2008 immer noch das größte. Etwas an Bedeutung gewonnen haben zwei Cluster, in denen häufiger eher problematische Themenaspekte angesprochen werden und die sich beide um den Bereich Recht und Sicherheit drehen. Sowohl das Cluster „The Dark Side“ der Digitalisierung als auch die Artikelgruppe, bei der es um die Konsequenzen politischen Handelns geht, haben etwas an Bedeutung zugelegt. Insgesamt kann man von einer gewissen Tendenz hin zu Beiträgen sprechen, in denen auch die Schattenseiten der Digitalisierung thematisiert werden. Dennoch bleibt es auch 2008 bei einer Berichterstattung, die digitale Technologien in einer Weise rahmt, dass ein überwiegend freundliches Bild entsteht.

An den Delta-Werten in Tabelle 4 ist zu erkennen, dass sich die Clusterstruktur auch intern nur geringfügig verändert hat. Gerade einmal bei vier der 85 Variablen-Clusterkombinationen sind signifikante Mittelwertunterschiede vorzufinden, und nur in drei Fällen $(<4 \%$ ) treten diese absoluten Veränderungen (Delta=0,3) etwas deutlicher hervor. In weiteren 12 Fällen (14\%) sind mittlere Veränderungen (Delta=0,2) zu beobachten. Bei den restlichen 82 Prozent konnten keine oder allenfalls geringfügige Veränderungen ermittelt werden.

Betrachtet man die Veränderungen in der Gesamtschau, dann sind jedoch Muster zu erkennen: Zum einen fällt auf, dass sich die Veränderungen auf zwei Cluster konzentrieren. Dabei handelt es sich um die Cluster Wirtschaftlicher Wettbewerb und Konsequenzen politischen Handelns. Zum anderen ist zu erkennen, dass sich - wenn man den 
Abbildung 1: Veränderung der Clustergrößen 2003/2008

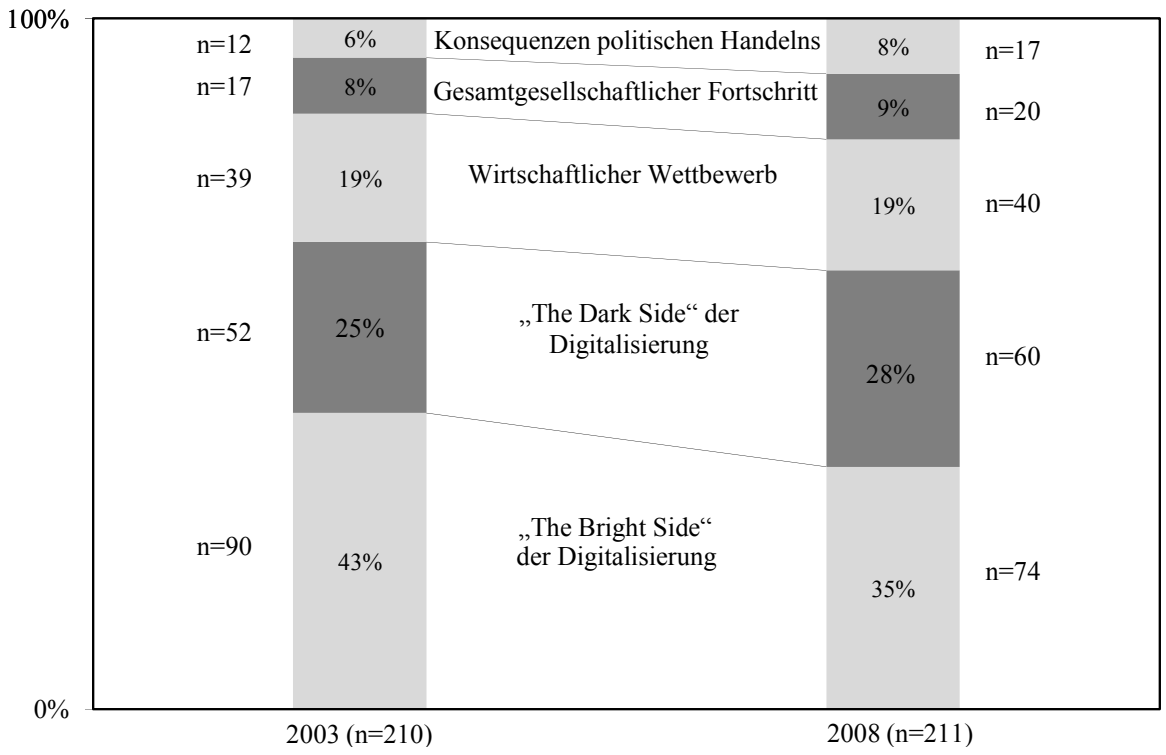

Vergleich auf die betroffenen Variablen fokussiert - die Veränderungen auf die wirtschaftsbezogenen Indikatoren konzentrieren: Insgesamt zeigt sich, dass den wirtschaftlichen Akteuren seltener die Urheberschaft für positive (wirtschaftliche) Folgen zugeschrieben wird. Im Frame Wirtschaftlicher Wettbewerb zeigt sich zudem, dass die Wirtschaft (neben der Gesellschaft) auch seltener als begünstigt und häufiger als geschädigt dargestellt wird. Fast gegenläufig stellt sich die Entwicklung bei den Konsequenzen politischen Handelns dar: In den Beiträgen dieses Clusters geht es nun seltener um geschädigte Wirtschaftsakteure.

Fasst man die Befunde der internen Clusterentwicklung zusammen, dann ist der dominierende Befund die Feststellung einer weitgehenden Stabilität. Die Veränderungen sind vor allem im wirtschaftlichen Bereich festzustellen. 2008 werden vermehrt negative Aspekte des Wettbewerbs in diesem Cluster thematisiert. Eine geringere Rolle spielt hingegen der mögliche und tatsächliche Nutzen, den die Aktivitäten der Wirtschaftsakteure hervorbringen.

\subsection{Zeitliche Veränderung der holistischen Frames}

Auch die mit den holistischen Frames ermittelten Ergebnisse verdeutlichen, dass sich zwischen den beiden Untersuchungszeiträumen nur geringfügige Unterschiede feststellen lassen und zwar sowohl hinsichtlich der Relevanz der acht Frames als auch bezüglich der einzelnen Ausprägungen der Frames (Tabelle 5).

Trotz dieser generellen Stabilität konnten dennoch einige bemerkenswerte Veränderungen im Detail festgestellt werden. Am deutlichsten fielen sie beim Technik-Frame aus: Die positive Ausprägung des Technik-Frames ging um 12 Prozentpunkte zurück, während die ambivalente Kategorie um 5 Prozentpunkte zulegte. Der Anteil der Artikel ganz ohne Technik-Frame wuchs gleichfalls um 7 Prozentpunkte. Neben dem Rückgang des positiven Technik-Frames sind auch nennenswerte Zuwächse beim Kontroverse- 
Tabelle 4: Mittelwerte (gesamt) und absolute Mittelwertsdifferenzen (2003/2008) der Frame-Elemente nach Cluster

\begin{tabular}{|c|c|c|c|c|c|c|c|c|c|c|c|c|}
\hline \multirow[t]{3}{*}{ Frameelemente } & \multicolumn{10}{|c|}{ Cluster } & & \\
\hline & \multicolumn{2}{|c|}{$\begin{array}{l}\text { "The Bright } \\
\text { Side" der } \\
\text { Digitali- } \\
\text { sierung } \\
(\mathrm{n}=164)\end{array}$} & \multicolumn{2}{|c|}{$\begin{array}{l}\text { "The Dark } \\
\text { Side" der } \\
\text { Digitali- } \\
\text { sierung } \\
(\mathrm{n}=112)\end{array}$} & \multicolumn{2}{|c|}{$\begin{array}{l}\text { Wirtschaft- } \\
\text { licher } \\
\text { Wettbewerb } \\
(\mathrm{n}=79)\end{array}$} & \multicolumn{2}{|c|}{$\begin{array}{l}\text { Gesamt- } \\
\text { geselllschaft- } \\
\text { licher } \\
\text { Fortschritt } \\
(\mathrm{n}=37)\end{array}$} & \multicolumn{2}{|c|}{$\begin{array}{c}\text { Konse- } \\
\text { quenzen } \\
\text { politischen } \\
\text { Handelns } \\
(\mathrm{n}=29)\end{array}$} & \multicolumn{2}{|c|}{$\begin{array}{r}\begin{array}{r}\text { Gesamt } \\
(\mathrm{n}=421) \\
\mathrm{MW}\end{array}\end{array}$} \\
\hline & MW & MW $\Delta$ & MW & MW $\Delta$ & MW & MW $\Delta$ & MW & MW $\Delta$ & MW & MW $\Delta$ & MW & $\Delta$ \\
\hline $\begin{array}{l}\text { Thema: Kultur, } \\
\text { Unterhaltung \& Freizeit }\end{array}$ & 0,5 & & 0,2 & $-0,2$ & 0,3 & $-0,1$ & 0,1 & & 0,1 & & 0,3 & $-0,1$ \\
\hline Thema: Wirtschaft & 0,0 & & 0,0 & & 0,8 & 0,2 & 0,0 & $-0,1$ & 0,0 & $-0,1$ & 0,2 & \\
\hline $\begin{array}{l}\text { Thema: Recht und } \\
\text { Sicherheit }\end{array}$ & 0,3 & & 0,7 & & 0,1 & & 0,1 & & 0,9 & $-0,1$ & 0,4 & \\
\hline Thema: Sonstiges & 0,0 & & 0,0 & & 0,0 & & 1,0 & 0,1 & 0,0 & $-0,1$ & 0,1 & \\
\hline $\begin{array}{l}\text { Problemdefinition des } \\
\text { Themas (Index, } \\
0=\text { negative Wertung, } \\
\text { Schaden, } 1=\text { positive } \\
\text { Wertung, Nutzen) }\end{array}$ & 0,8 & & 0,4 & & 0,6 & $-0,1$ & 0,7 & & 0,3 & & 0,6 & \\
\hline $\begin{array}{l}\text { Begünstigter d. Nutzens: } \\
\text { Wirtschaft }\end{array}$ & 0,1 & $-0,1^{*}$ & 0,1 & & 0,5 & $-0,2$ & 0,2 & 0,1 & 0,2 & & 0,2 & $-0,1$ \\
\hline $\begin{array}{l}\text { Begünstigter d. Nutzens: } \\
\text { Gesellschaft }\end{array}$ & 0,9 & & 0,3 & & 0,5 & $-0,2 *$ & 0,7 & $-0,2$ & 0,3 & 0,2 & 0,6 & $-0,1$ \\
\hline Geschädigter: Wirtschaft & 0,0 & & 0,2 & $-0,1$ & 0,5 & 0,2 & 0,1 & $-0,1$ & 0,2 & $-0,3$ & 0,2 & \\
\hline $\begin{array}{l}\text { Geschädigter: } \\
\text { Gesellschaft }\end{array}$ & 0,0 & & 0,5 & 0,1 & 0,2 & $-0,1$ & 0,4 & 0,1 & 0,3 & 0,2 & 0,2 & 0,1 \\
\hline $\begin{array}{l}\text { Verantwortlich f. Nutzen: } \\
\text { Politik }\end{array}$ & 0,2 & & 0,0 & & 0,0 & & 0,2 & & 0,6 & 0,2 & 0,1 & \\
\hline $\begin{array}{l}\text { Verantwortlich f. Nutzen: } \\
\text { Wirtschaft }\end{array}$ & 0,4 & $-0,1$ & 0,1 & & 0,7 & $-0,3 *$ & 0,5 & $-0,3$ & 0,1 & $-0,2$ & 0,4 & $-0,2$ \\
\hline $\begin{array}{l}\text { Verantwortlich f. } \\
\text { Nutzen: Medien \& } \\
\text { Gesellschaft }\end{array}$ & 0,2 & $0,2^{*}$ & 0,1 & & 0,0 & & 0,1 & & 0,0 & & 0,1 & 0,1 \\
\hline $\begin{array}{l}\text { Verantwortlich f. } \\
\text { Schaden: Politik }\end{array}$ & 0,0 & & 0,0 & & 0,0 & & 0,1 & & 1,0 & & 0,1 & \\
\hline $\begin{array}{l}\text { Verantwortlich } \mathrm{f} \text {. } \\
\text { Schaden: Wirtschaft }\end{array}$ & 0,0 & & 0,2 & $-0,1$ & 0,5 & $-0,2$ & 0,1 & $-0,1$ & 0,0 & 0,1 & 0,2 & $-0,1$ \\
\hline $\begin{array}{l}\text { Verantwortlich f. } \\
\text { Schaden: Medien \& } \\
\text { Gesellschaft }\end{array}$ & 0,0 & & 0,3 & 0,1 & 0,1 & 0,1 & 0,1 & & 0,0 & $-0,1$ & 0,1 & \\
\hline $\begin{array}{l}\text { Prognose }(0=\text { negativ, } \\
1=\text { positiv })\end{array}$ & 0,7 & & 0,4 & & 0,6 & & 0,6 & & 0,5 & 0,1 & 0,6 & \\
\hline Handlungsempfehlung & 0,2 & & 0,3 & & 0,2 & & 0,1 & & 0,2 & & 0,2 & \\
\hline
\end{tabular}

${ }^{1}$ MW $\Delta=$ absolute Differenz der Mittelwerte der Frameelemente zwischen den Clustern der Jahre 2003 und 2008. Werte mit negativem Vorzeichen bedeuten eine Abnahme, Werte mit positivem Vorzeichen stehen für eine Zunahme.

${ }^{2}$ Soweit nicht ausgewiesen gelten für die Frameelemente folgende Ausprägungen: $0=$ nicht genannt, $1=$ genannt.

${ }^{3}$ Prüfung auf signifikante Mittelwertsunterschiede mittels t-Test für unabhängige Stichproben. Es gilt dabei: * p $<.05$.

${ }^{4}$ Auf den Ausweis von MW $\Delta=0$ wurde verzichtet. 
Frame (7 Prozentpunkte) und beim Popularisierungs-Frame (6 Prozentpunkte) zu verzeichnen. Diese Veränderungen bekommen ihre Relevanz nicht durch ihre absolute Höhe, sondern dadurch, dass diese Frames 2003 fast gar keine Rolle gespielt haben und sich deswegen die Prozentzahlen - obwohl es sich nur um relativ kleine Zuwächse handelt - mehr als verdoppelt haben. Alles in allem zeichnet sich auch bei dieser Operationalisierung der Frames eine gewisse Verdüsterung des Bildes ab: weniger positive Technikrahmung und mehr Konflikte.

Tabelle 5: Anteile der holistischen Medien-Frames im Jabresvergleich 2003/2008*

\begin{tabular}{|c|c|c|c|c|c|c|c|c|}
\hline & \multicolumn{2}{|c|}{ nicht vorhanden } & \multicolumn{2}{|c|}{ positiv dargestellt } & \multicolumn{2}{|c|}{$\begin{array}{l}\text { positiv und negativ } \\
\text { dargestellt }\end{array}$} & \multicolumn{2}{|c|}{ negativ dargestellt } \\
\hline & 2003 & 2008 & 2003 & 2008 & 2003 & 2008 & 2003 & 2008 \\
\hline Technik-Frame & 60 & 67 & 31 & 19 & 7 & 12 & 2 & 2 \\
\hline Gesellschafts-Frame & 91 & 86 & 4 & 6 & 3 & 4 & 2 & 4 \\
\hline Ökonomie-Frame & 85 & 78 & 5 & 9 & 5 & 9 & 5 & 5 \\
\hline \multirow[t]{3}{*}{ Politik-Frame } & 83 & 85 & 8 & 5 & 5 & 6 & 5 & 4 \\
\hline & \multicolumn{4}{|c|}{ nicht vorhanden } & \multicolumn{4}{|c|}{ vorhanden } \\
\hline & \multicolumn{2}{|c|}{2003} & \multicolumn{2}{|c|}{2008} & \multicolumn{2}{|c|}{2003} & \multicolumn{2}{|c|}{2008} \\
\hline Popularisierungs-Frame & \multicolumn{2}{|c|}{95} & \multicolumn{2}{|c|}{89} & \multicolumn{2}{|c|}{5} & \multicolumn{2}{|c|}{11} \\
\hline Orientierungs-Frame & \multicolumn{2}{|c|}{94} & \multicolumn{2}{|c|}{92} & \multicolumn{2}{|c|}{6} & \multicolumn{2}{|c|}{8} \\
\hline Kontroverse-Frame & \multicolumn{2}{|c|}{96} & \multicolumn{2}{|c|}{89} & \multicolumn{2}{|c|}{4} & \multicolumn{2}{|c|}{11} \\
\hline Skandal-Frame & \multicolumn{2}{|c|}{99} & \multicolumn{2}{|c|}{97} & \multicolumn{2}{|c|}{1} & \multicolumn{2}{|c|}{3} \\
\hline
\end{tabular}

* 2003 n=210, 2008 n=211; Angaben in Zeilenprozent nach Jahr

Die Ergebnisse, die mit den holistischen Frames erzielt wurden, verdeutlichen somit ebenfalls, dass Stabilität der dominierende Befund ist, auch wenn bei einzelnen Frames und ihren Ausprägungen durchaus interpretierbare Veränderungen festzustellen sind. Angesichts der vorliegenden Resultate kann die erste Forschungsfrage dahingehend beantwortet werden, dass beide Operationalisierungsvorschläge für Medien-Frames zu ähnlichen Befunden geführt haben: Die empirischen Ergebnisse in beiden Zeiträumen sind strukturell weitgehend identisch. Die wenigen Unterschiede, die zwischen den Untersuchungszeitpunkten festzustellen waren, deuten auf eine tendenzielle Eintrübung des Images digitaler Technologie.

\subsection{Holistische und clusteranalytisch ermittelte Frames im Vergleich}

Da die bisher dargestellten Befunde verdeutlichen, dass die Unterschiede zwischen den Jahren nur geringfügig ausfallen, können die nun folgenden Analysen - welche die zweite, methodenorientierte Forschungsfrage beantworten sollen - mit dem Gesamtsample durchgeführt werden. Um den Vergleich zwischen den holistisch erfassten und den clusteranalytisch ermittelten Frames durchzuführen, wurden jeweils Kreuztabellen berechnet. Für jeden der fünf Cluster-Frames wurde ermittelt, wie sich bei ihnen die Ausprägungen der acht holistischen Frames jeweils verteilen (Tabellen 6-10).

Die Kennzeichnung des größten Clusters mit dem Label „Bright Side“ wird durch die Befunde der holistischen Frames bekräftigt. Negative Ausprägungen der ersten vier Frames kommen fast gar nicht vor, auch Skandal- und Kontroverse-Frames spielen in den Beiträgen dieses Clusters keine Rolle. Eine zentrale Rolle spielt hingegen der positive Technik-Frame. Ebenso spielt der Popularisierungs-Frame eine überdurchschnittliche 
Tabelle 6: Ausprägung der holistischen Medien-Frames im Cluster „The Bright Side“ der Digitalisierung ( $n=164$, Angaben in Zeilenprozent)

\begin{tabular}{|c|c|c|c|c|}
\hline & nicht vorhanden & $\begin{array}{c}\text { positiv } \\
\text { dargestellt }\end{array}$ & $\begin{array}{l}\text { positiv und } \\
\text { negativ } \\
\text { dargestellt }\end{array}$ & $\begin{array}{c}\text { negativ } \\
\text { dargestellt }\end{array}$ \\
\hline Technik-Frame & 46,3 & 42,7 & 9,8 & 1,2 \\
\hline Gesellschafts-Frame & 89,6 & 8,5 & 1,8 & 0,0 \\
\hline Ökonomie-Frame & 92,1 & 4,9 & 2,4 & 0,6 \\
\hline Politik-Frame & 87,8 & 7,9 & 4,3 & 0,0 \\
\hline & \multicolumn{2}{|c|}{ nicht vorhanden } & \multicolumn{2}{|c|}{ vorhanden } \\
\hline Popularisierungs-Frame & \multicolumn{2}{|c|}{84,8} & \multicolumn{2}{|c|}{15,2} \\
\hline Orientierungs-Frame & \multicolumn{2}{|c|}{95,7} & \multicolumn{2}{|c|}{4,3} \\
\hline Kontroverse-Frame & \multicolumn{2}{|c|}{98,8} & \multicolumn{2}{|c|}{1,2} \\
\hline Skandal-Frame & \multicolumn{2}{|c|}{100,0} & \multicolumn{2}{|c|}{0,0} \\
\hline
\end{tabular}

Tabelle 7: Ausprägung der holistischen Medien-Frames im Cluster „The Dark Side“ der Digitalisierung ( $n=112$, Angaben in Zeilenprozent)

\begin{tabular}{|c|c|c|c|c|}
\hline & nicht vorhanden & $\begin{array}{c}\text { positiv } \\
\text { dargestellt }\end{array}$ & $\begin{array}{l}\text { positiv und } \\
\text { negativ } \\
\text { dargestellt }\end{array}$ & $\begin{array}{c}\text { negativ } \\
\text { dargestellt }\end{array}$ \\
\hline Technik-Frame & 81,2 & 8,0 & 7,1 & 3,6 \\
\hline Gesellschafts-Frame & 80,4 & 0,9 & 8,9 & 9,8 \\
\hline Ökonomie-Frame & 89,3 & 1,8 & 1,8 & 7,1 \\
\hline Politik-Frame & 81,2 & 7,1 & 4,5 & 7,1 \\
\hline & \multicolumn{2}{|c|}{ nicht vorhanden } & \multicolumn{2}{|c|}{ vorhanden } \\
\hline Popularisierungs-Frame & \multicolumn{2}{|c|}{98,2} & \multicolumn{2}{|c|}{1,8} \\
\hline Orientierungs-Frame & \multicolumn{2}{|c|}{89,3} & \multicolumn{2}{|c|}{10,7} \\
\hline Kontroverse-Frame & \multicolumn{2}{|c|}{90,2} & \multicolumn{2}{|c|}{9,8} \\
\hline Skandal-Frame & \multicolumn{2}{|c|}{92,9} & \multicolumn{2}{|c|}{7,1} \\
\hline
\end{tabular}

Rolle, der anzeigt, dass in einem Beitrag die technischen Errungenschaften für ein breites Publikum verständlich aufbereitet werden. Alle anderen Frames kommen eher unterdurchschnittlich häufig vor und wenn, dann überwiegend in positiven Zusammenhängen.

Genau entgegengesetzt zu der Verteilung der holistischen Medien-Frames in Tabelle 6 verhält es sich mit dem „Dark Side“-Frame (Tabelle 7). Überdurchschnittlich häufig findet man die negativen Ausprägungen der ersten vier Frames in den Artikeln dieses Clusters und selten nur die positiven Ausprägungen. Vor allem ein positiver TechnikFrame wird hier nur sehr selten herangezogen. Vergleichsweise oft wird hingegen das Thema Digitalisierung mit Skandal-, Kontroverse- und Orientierungs-Frames gerahmt.

Eine große Konsistenz der Befunde mit den holistischen Frames ist auch beim Cluster Wirtschaftlicher Wettbewerb festzustellen (Tabelle 8), denn hier dominiert der Ökonomie-Frame mit seinen verschiedenen Varianten: Den größten Anteil hat mit über 25 Prozent die mittlere Ausprägung des Frames, die sowohl positive als auch negative Effekte beinhaltet. Hier wird der wirtschaftliche Wettbewerb besonders deutlich. Aber auch die nur positive $(19 \%)$ und die nur negative $(11 \%)$ Darstellung ökonomischer 
Tabelle 8: Ausprägung der holistischen Medien-Frames im Cluster Wirtschaftlicher Wettbewerb ( $n=79$, Angaben in Zeilenprozent)

\begin{tabular}{|c|c|c|c|c|}
\hline & nicht vorhanden & $\begin{array}{c}\text { positiv } \\
\text { dargestellt }\end{array}$ & $\begin{array}{l}\text { positiv und } \\
\text { negativ } \\
\text { dargestellt }\end{array}$ & $\begin{array}{c}\text { negativ } \\
\text { dargestellt }\end{array}$ \\
\hline Technik-Frame & 73,4 & 12,7 & 13,9 & 0,0 \\
\hline Gesellschafts-Frame & 93,7 & 3,8 & 2,5 & 0,0 \\
\hline Ökonomie-Frame & 44,3 & 19,0 & 25,3 & 11,4 \\
\hline Politik-Frame & 94,9 & 1,3 & 2,5 & 1,3 \\
\hline & \multicolumn{2}{|c|}{ nicht vorhanden } & \multicolumn{2}{|c|}{ vorhanden } \\
\hline Popularisierungs-Frame & \multicolumn{2}{|c|}{96,2} & \multicolumn{2}{|c|}{3,8} \\
\hline Orientierungs-Frame & \multicolumn{2}{|c|}{92,4} & \multicolumn{2}{|c|}{7,6} \\
\hline Kontroverse-Frame & \multicolumn{2}{|c|}{88,6} & \multicolumn{2}{|c|}{11,4} \\
\hline Skandal-Frame & \multicolumn{2}{|c|}{100,0} & \multicolumn{2}{|c|}{0,0} \\
\hline
\end{tabular}

Tabelle 9: Ausprägung der holistischen Medien-Frames im Cluster Gesamtgesellschaftlicher Fortschritt ( $n=37$, Angaben in Zeilenprozent)

\begin{tabular}{|c|c|c|c|c|}
\hline & nicht vorhanden & $\begin{array}{c}\text { positiv } \\
\text { dargestellt }\end{array}$ & $\begin{array}{l}\text { positiv und } \\
\text { negativ } \\
\text { dargestellt }\end{array}$ & $\begin{array}{c}\text { negativ } \\
\text { dargestellt }\end{array}$ \\
\hline Technik-Frame & 48,6 & 37,8 & 8,1 & 5,4 \\
\hline Gesellschafts-Frame & 89,2 & 8,1 & 0,0 & 2,7 \\
\hline Ökonomie-Frame & 89,2 & 8,1 & 2,7 & 0,0 \\
\hline Politik-Frame & 97,3 & 0,0 & 2,7 & 0,0 \\
\hline & \multicolumn{2}{|c|}{ nicht vorhanden } & \multicolumn{2}{|c|}{ vorhanden } \\
\hline Popularisierungs-Frame & \multicolumn{2}{|c|}{86,5} & \multicolumn{2}{|c|}{13,5} \\
\hline Orientierungs-Frame & \multicolumn{2}{|c|}{89,2} & \multicolumn{2}{|c|}{10,8} \\
\hline Kontroverse-Frame & \multicolumn{2}{|c|}{91,9} & \multicolumn{2}{|c|}{8,1} \\
\hline Skandal-Frame & \multicolumn{2}{|c|}{100,0} & \multicolumn{2}{|c|}{0,0} \\
\hline
\end{tabular}

Aspekte der Digitalisierung sind in diesem Cluster häufig vertreten. Bemerkenswert ist weiterhin, dass zwar der Kontroverse-Frame mit gut 11 Prozent stark ausgeprägt ist, dass aber andererseits in diesem Cluster weder in Bezug auf die Technik noch hinsichtlich der gesellschaftlichen Konsequenzen negative Ausprägungen der entsprechenden Frames (Technik/Gesellschaft) festzustellen sind. Außerwirtschaftliche negative Aspekte spielen in diesem Frame somit keine Rolle. Dass es in diesem Cluster mehr um Konkurrenz als um Negativismus geht, zeigt sich auch daran, dass der Skandal-Frame nicht ein einziges Mal vorkommt.

Gleichermaßen konnte eine hohe Übereinstimmung zwischen dem Cluster Gesamtgesellschaftlicher Fortschritt und den holistischen Medien-Frames festgestellt werden. Tabelle 9 zeigt, dass in diesem Cluster wiederum der Technik-Frame, aber auch der Popularisierungs- und der Orientierungs-Frame stärker vertreten sind, wobei der Technik-Frame mit knapp 38 Prozent eine klar positive Färbung erhalten hat. Der PolitikFrame ist allerdings etwas schwächer ausgeprägt, als es die Analyse des Frames „Gesamtgesellschaftlicher Fortschritt" anhand der Einzelvariablen hätte erwarten lassen. Da der Kontroverse-Frame zudem nur durchschnittlich, die negativen Ausprägungen der 
Tabelle 10: Ausprägung der holistischen Medien-Frames im Cluster Konsequenzen politischen Handelns ( $n=29$, Angaben in Zeilenprozent)

\begin{tabular}{|c|c|c|c|c|}
\hline & nicht vorhanden & $\begin{array}{c}\text { positiv } \\
\text { dargestellt }\end{array}$ & $\begin{array}{l}\text { positiv und } \\
\text { negativ } \\
\text { dargestellt }\end{array}$ & $\begin{array}{c}\text { negativ } \\
\text { dargestellt }\end{array}$ \\
\hline Technik-Frame & 86,2 & 3,4 & 6,9 & 3,4 \\
\hline Gesellschafts-Frame & 96,6 & 0,0 & 0,0 & 3,4 \\
\hline Ökonomie-Frame & 79,3 & 6,9 & 6,9 & 6,9 \\
\hline Politik-Frame & 24,1 & 17,2 & 27,6 & 31,0 \\
\hline & \multicolumn{2}{|c|}{ nicht vorhanden } & \multicolumn{2}{|c|}{ vorhanden } \\
\hline Popularisierungs-Frame & \multicolumn{2}{|c|}{100,0} & \multicolumn{2}{|c|}{0,0} \\
\hline Orientierungs-Frame & \multicolumn{2}{|c|}{100,0} & \multicolumn{2}{|c|}{0,0} \\
\hline Kontroverse-Frame & \multicolumn{2}{|c|}{72,4} & \multicolumn{2}{|c|}{27,6} \\
\hline Skandal-Frame & \multicolumn{2}{|c|}{100,0} & \multicolumn{2}{|c|}{0,0} \\
\hline
\end{tabular}

anderen Frames eher unterdurchschnittlich und der Skandal-Frame sogar überhaupt nicht vorhanden sind, kann diesem Cluster eine überwiegend positive Darstellung der Digitalisierung attestiert werden, was wiederum die Bezeichnung „Fortschritt“ rechtfertigt.

Das Cluster Konsequenzen politischen Handelns in Tabelle 10 ist ebenfalls sehr prägnant durch spezifische Ausprägungen der holistischen Medien-Frames gekennzeichnet. Hier ist der Politik-Frame sehr ausgeprägt, wobei die negative Darstellung mit 31 Prozent den höchsten Anteil hat, dicht gefolgt von einer tendenziell neutralen Darstellung (positiv und negativ) mit knapp 28 Prozent. In dieses Bild passt auch, dass sowohl beim Technik-Frame als auch beim Ökonomie-Frame der Anteil positiver und negativer Darstellungen jeweils gleich ist, was ebenfalls auf eine Berichterstattung schließen lässt, die ganz unterschiedliche Konsequenzen der Digitalisierung mit variierenden Wertigkeiten thematisiert. Die Werte, die allerdings am stärksten ins Auge springen, betreffen die Frames Popularisierung, Orientierung, Skandal und Kontroverse. Während die drei ersten Frames in diesem Cluster überhaupt nicht vorhanden sind, schlägt der KontroverseFrame mit fast 28 Prozent stark zu Buche. Durch den relativ hohen Anteil von Kontroversen tritt der Charakter politischer Auseinandersetzung, den die Beiträge in diesem Cluster aufweisen, deutlich zu Tage, was jedoch von den Rezipienten häufig als negativ wahrgenommen wird.

Alles in allem bestätigt die Gegenüberstellung der zwei methodisch unterschiedlich ermittelten Frame-Typen, dass auch in diesem Fall mit beiden Methoden Ergebnisse erzielt werden, die hinsichtlich ihrer Grundtendenz durchaus vergleichbar erscheinen und sich damit auch gegenseitig validieren.

\section{Fazit}

Ziel dieses Beitrags war es, zwei Forschungsfragen bezüglich der Darstellung des Themas Digitalisierung und Gesellschaft durch die Medien zu beantworten.

Die erste Forschungsfrage - Wie wird über das Thema Digitalisierung berichtet und welche Veränderungen lassen sich zwischen den Jahren 2003 und 2008 feststellen? kann dahingehend beantwortet werden, dass die Befunde eine weitgehende Stabilität der Art und Weise der Darstellung zeigen. Die empirischen Ergebnisse in beiden Zeiträumen sind strukturell überwiegend identisch. Die wenigen feststellbaren Unterschiede deuten 
auf eine tendenzielle Eintrübung des Images digitaler Technologie hin. Nichtsdestotrotz wird von den Medien mehrheitlich ein eher positives Bild der Digitalisierung gezeichnet. Allerdings ist dabei zu berücksichtigen, dass dies nicht für alle Bereiche gilt. Sobald nicht nur der eher konfliktfreie Unterhaltungsbereich im Mittelpunkt steht, sondern politische Aspekte oder Fragen von Recht und Sicherheit thematisiert werden, wandelt sich dieses Bild stark. Deutlich wird in jedem Fall, dass das Thema Digitalisierung zwar als ein Bereich der Technikberichterstattung begriffen werden kann, jedoch nicht nur als Teil der Risiko- und Wissenschaftskommunikation konzeptionalisiert werden darf, wie dies in der Forschung zur Technikberichterstattung häufig geschieht. Dies bestätigt wiederum den hier gewählten erweiterten Zugang zum Thema Digitalisierung, in dem erstens nicht einzelne Produkte, Serviceleistungen oder Anwendungen untersucht wurden und zweitens insbesondere anhand der Wahl der holistischen Frames alle Bereiche der Gesellschaft bezüglich der Technikberichterstattung systematisch integriert wurden.

Bezüglich der zweiten, methodisch orientierten Forschungsfrage - Welche Unterschiede und Gemeinsamkeiten in den Ergebnissen sind auf unterschiedliche Operationalisierungen von Medien-Frames zurückzuführen? - kann attestiert werden, dass mit beiden Methoden Ergebnisse erzielt werden, die hinsichtlich ihrer Grundtendenz durchaus vergleichbar erscheinen.

Der Einwand, clusteranalytische Verfahren würden je nach Studie immer neue empirische Frames abbilden, aber keinen theoretischen Beitrag zur Framing-Forschung leisten, ist hier durch den validierenden Einsatz dieser Auswertungsmethode relativiert worden (Scheufele/Scheufele 2009). Auch die grundlegenden Zweifel an der Machbarkeit der Ermittlung holistischer Frames scheinen angesichts der vorliegenden Ergebnisse übertrieben. Vielmehr zeigen die Befunde, dass durch die Kombination beider Verfahren dichtere Beschreibungen der Berichterstattungseigenschaften und -tendenzen möglich sind. Konkret heißt dies, dass rekonstruktive Frames insofern nicht nur als Ergänzung der holistischen Frames gesehen werden können, sondern auch als eine Verdichtung letzterer. Bezogen auf die Beantwortung unserer zweiten Forschungsfrage lässt sich somit festhalten, dass die rekonstruktive Frame-Ermittlung insofern holistische Frames verdichtet, als die kodierten Variablen oder Frame-Elemente Detail-Aspekte von holistischen Deutungsmustern abdecken. Letztere stellen dabei eine generelle Einfärbung oder Tendenz des Medientextes dar. Die rekonstruktiven Frames bilden demnach genauere Beschreibungen dieser Einfärbung ab.

Kritisch anzumerken ist jedoch, dass es nicht ausgeschlossen werden kann, dass die gute Passfähigkeit der beiden unterschiedlichen Frame-Messungen zumindest teilweise darauf zurückzuführen ist, dass beide Kodierungen jeweils von den gleichen Kodierern durchgeführt wurden. Um diesen Einfluss bei weiteren Erhebungen zu minimieren, wäre es sinnvoll, die Kodierer in zwei Teams aufzuteilen, die unabhängig voneinander jeweils die beiden unterschiedlichen Kodierungen von Frames durchführen.

\section{Ausblick}

Die vorgelegten Analysen sind der erste Schritt bei der Auswertung der vorliegenden Daten. In weiteren Auswertungen wird unter anderem eine Gegenüberstellung der Darstellungsweise in den unterschiedlichen Medien, also zum Beispiel konservativ-orientierte vs. links-orientierte Medien erfolgen. Dies könnte sich - nicht zuletzt vor dem Hintergrund der internen Entwicklung des Clusters Wirtschaftlicher Wettbewerb - als aufschlussreich erweisen: 2008 werden vermehrt negative Aspekte des Wettbewerbs in diesem Cluster thematisiert, dass die Wirtschaftsakteure hingegen Nutzen stiften, spielt eine nicht mehr ganz so große Rolle. Interessant dürfte weiterhin sein, ob sich die Medien 
hinsichtlich der Konflikte, die sich in den Bereichen „politsche Konsequenzen“ und „Recht und Sicherheit“ abzeichnen, bereits positioniert haben. Eine solche Positionierung würde dafür sprechen, dass die Digitalisierung der Gesellschaft zumindest mittelfristig ein größeres Konfliktpotenzial birgt, als es bislang den Anschein hat.

In methodischer Hinsicht sollte insbesondere die in Abschnitt 2 erwähnte computergestützte Frame-Analyse weiterentwickelt werden. Bereits bei Entman (1993) ist eine starke Betonung der Bedeutung von Sprache zu finden, indem er den rekurrierenden und konsistenten Einsatz von bestimmten Wörtern oder Satzstrukturen hervorhebt. Hier bietet sich eine Verknüpfung mit linguistischen Ansätzen, insbesondere der quantitativen Diskursanalyse, an. Während dort konkret rekurrierende Wörter (Lexeme) und Wortstellungen im Kontext von bestimmten anderen Wörtern (Kookkurrenz) herausgearbeitet werden, gehen Framing-Analysen selten in diese lexikalische Tiefe. Jedoch gibt es Übereinstimmungen, beispielsweise was Fragen nach den konkreten Sprechern/ Akteuren und deren Positionen sowie entscheidenden Ereignissen und Themen-/Subthemenbestimmung angeht. So finden sich bei Miller (1997) Inhaltsanalysen, deren Methode einerseits den statistischen Techniken der Korpuslinguistik (die auch in der quantitativen Diskursanalyse angewendet werden) angelehnt sind, und andererseits von der Framing-Definition von Entman ausgehen. Der Autor nennt dieses Vorgehen FrameMapping: "Frame mapping method provides a rigorous way to investigate frames as Entman explicates them. It provides for ascertaining an appropriate set of key words used by stakeholders to articulate their frames for an issue and for determining patterns of key word use“ (Miller 1997, S. 368). Matthes (2008, S. 161) spricht im Hinblick auf die rekonstruktive Frame-Ermittlung (bei ihm „manuell-dimensionsreduzierend“ genannt) nicht von „key words“, sondern von Textmustern, die sich letztendlich aber durch eine bestimmte Wortwahl und syntaktische Wortstellung rekonstruieren lassen müssten. Eine solche computerbasierte Vorgehensweise bietet die Chance, die Probleme, die mit einer validen und reliablen Identifizierung von Frames durch Kodierer verbunden sind, zu umgehen. Ob sie aber tatsächlich geeignet ist, sinnvoll interpretierbare Medien-Frames zu identifizieren, dafür bedarf es noch weiterer empirischer Erfahrung.

\section{Literatur}

Bacher, J. (2002): Clusteranalyse. Anwendungsorientierte Einführung. München: Oldenbourg.

Backhaus, K., Erichson, B., Plinke, W., \& Weiber, R. (2006): Multivariate Analysemethoden: Eine anwendungsorientierte Einführung. Berlin: Springer.

Beck, K., \& Vowe, G. (1995): Multimedia aus der Sicht der Medien. Argumentationsmuster und Sichtweisen in der medialen Konstruktion. Rundfunk und Fernsehen, 43(4), 549-563.

Bonfadelli, H. (2002): Medieninhaltsforschung. Konstanz: UVK.

Dahinden, U., \& Schanne, M. (2009): Wissenschafts- und Risikokommunikation. In D. Süss (Hrg.), Medienrealitäten. Konstanz: UVK, S. 69-88.

D’Angelo, P., \& Kuypers, J. A. (2009): Doing News Framing Analysis. Empirical and Theoretical Perspectives. New York: Routledge.

Eikmann, T., \& Herr, C. (2008): Mobilfunkdiskurse und die problematische Rolle der Medien. Technikfolgenabschätzung - Theorie und Praxis, 17(3), 57-66. doi: 10.1002/ckon.200890036.

Entman, R. M. (1993): Framing: Toward Clarification of a Fractured Paradigm. Journal of Communication, 43(4), 51-58.

Entman, R. M., Matthes, J., \& Pellicano, L. (2009): Nature, Sources and Effects of News Framing. In K. Wahl-Jorgensen \& T. Hanitzsch (Hrsg.), Handbook of Journalism Studies. Mahwah NJ: Lawrence Erlbaum, S. 175-190.

Giesecke, S. (2003): Technikakzeptanz durch Nutzerintegration? Beiträge zur Innovations- und Technikanalyse. Informationstechnik. Teltow: VDI/VDE. 
Hansen, J. (1995): Wie man die Umwelt selbst erlebt und wie in den Medien. In G. de Haan (Hrsg.), Umweltbewußtsein und Massenmedien. Berlin: Akademie Verlag, S. 103-113.

Kelly, J. P. (2009): Not so Revolutionary After All: The Role of Reinforcing Frames in US Magazine Discourse about Microcomputers. New Media \& Society, 11(1-2), 31-52.

Kepplinger, H. M. (1989): Künstliche Horizonte. Frankfurt/New York: Campus.

Kepplinger, H. M. (1992): Ereignismanagement. Wirklichkeit der Massenmedien. Osnabrück: Fromm.

Kepplinger, H. M. (1993): Technik-Kritik in den Medien. In H. Bonfadelli \& W. A. Meier (Hrsg.), Krieg, Aids, Katastrophen... Gegenwartsprobleme als Herausforderung der Publizistikwissenschaft. Konstanz: UVK, S. 193-211.

Kessel, T., Gerneth, M., \& Wolf, M. (2009): Zukunft und Zukunftsfähigkeit der Informations- und Kommunikationstechnologien und Medien. Internationale Delphi-Studie 2030. Münchner Kreis e. V., EICT GmbH, Deutsche Telekom AG, TNS Infratest GmbH. www.tns-infratest.com/presse/Zukunft_IKT.asp [06.10.2010].

Kohring, M., \& Matthes, J. (2002): The Face(t)s of Biotech in the Nineties: How the German Press Framed Modern Biotechnology. Public Understanding of Science, 11(2), 143-154.

Kolb, S. (2005): Mediale Thematisierung in Zyklen. Theoretischer Entwurf und empirische Anwendung. Köln: Halem.

Matthes, J. (2008): Medien-Frames inhaltsanalytisch (be)greifen. Eine Analyse von 135 nationalen und internationalen Fachzeitschriftenaufsätzen, 1990-2005. In J. Matthes, W. Wirth, G. Daschmann, \& A. Fahr (Hrsg.), Die Brücke zwischen Theorie und Empirie: Operationalisierung, Messung und Validierung in der Kommunikationswissenschaft. Köln: Halem, S. 28-47.

Matthes, J., \& Kohring, M. (2004): Die empirische Erfassung von Medien-Frames. Medien \& Kommunikationswissenschaft, 52(1), 56-75.

Maurer, M., \& Reinemann, C. (2006): Medieninhalte. Wiesbaden: VS Verlag.

Miller, M. M. (1997): Frame Mapping and Analysis of News Coverage of Contentious Issues. Social Science Computer Review, 15(4), 367-378.

Negroponte N. (1995): Being digital. NewYork: Knopf.

Noelle-Neumann, E., Kepplinger, H. M., \& Donsbach, W. (1999): Kampa. Meinungsklima und Medienwirkung im Bundestagswahlkampf 1998. Freiburg/München: Karl Alber.

Peters, H. P. (1994): Wissenschaftliche Experten in der öffentlichen Kommunikation über Technik, Umwelt und Risiken. In F. Neidhardt (Hrsg.), Öffentlichkeit, öffentliche Meinung, soziale Bewegungen. Opladen, S. 162-190.

Rössler, P. (2001): Between Online Heaven and Cyberhell: The Framing of 'The Internet' by Traditional Media Coverage in Germany. New Media \& Society, 3(1), 49-66.

Scheufele, B. (2003): Frames - Framing - Framing-Effekte. Theoretische und methodische Grundlegung des Framingansatzes sowie empirische Befunde zur Nachrichtenproduktion. Wiesbaden: Westdeutscher Verlag.

Scheufele, B. \& Brosius, H.-B. (1999): The Frame Remains the Same? Stabilität und Kontinuität journalistischer Selektionskriterien am Beispiel der Berichterstattung über Anschläge auf Ausländer und Asylbewerber. Rundfunk und Fernsehen, 47(3), 409-432.

Scheufele, B. T. \& Scheufele, D. A. (2009): Of Spreading Activation, Applicability, and Schemas. Conceptual Distinctions and their Operational Implications for Measuring Frames and Framing Effects. In P. D’Angelo \& J. A. Kuypers (Hrsg.), Doing News Framing Analysis. Empirical and Theoretical Perspectives: New York: Routledge, S. 110-134.

Scheufele, D. A. (1999): Framing as a Theory of Media Effects. Journal of Communication, 49(1), 103-122. 